\title{
Delivering High Intensity Proton Beam: Lessons for the Next Beam Generations
}

\section{Sam Childress ${ }^{1}$}

Fermilab

Batavia, IL. 60510, U.S.A.

E-mail: childressefnal.gov

The current generation of high intensity proton beams for neutrino oscillation experiments has led to development of similar techniques for controlling the different intense beams of several hundred kilowatts beam power. We report on these features, and the suitability for use with next generation proton beams in the megawatt regime.

10th International Workshop on Neutrino Factories, Super beams and Beta beams

Valencia, Spain

30 June-05 July, 2008

\footnotetext{
$1 \quad$ Speaker
} 


\section{Introduction}

Neutrino experiments to measure the effect of oscillations, in which neutrinos change from one type into another, are currently key components of the physics research programs at accelerator laboratories in Japan, the United States and Europe. To generate the intense fluxes of neutrinos needed, these experiments must target the most intense proton beams achievable, with beam powers measured in hundreds of kilowatts $(\mathrm{kW})$. These beams share some key design features and constraints, which are discussed in this paper. We also consider some important lessons for the next generation of these beams, with proton beam power reaching the megawatt (MW) regime.

\section{Key Proton Beam Cnsiderations}

The most compelling feature for several hundred $\mathrm{kW}$ proton beams is that they can damage most materials very quickly. A few seconds or even one cycle of mis-steered beam can readily disable most components. Besides prevention of accidental beam loss, significant limits on normal operation proton beam transport loss are also needed. Fractional beam loss limits of a few $\times 10^{-5}$ of the beam are desirable to provide component residual radioactivity levels enabling efficient maintenance and repair interventions. Another consideraiton is that very large numbers of integrated protons on target are needed to produce the neutrino fluxes for precision neutrino oscillation experiments. Typical experiment needs for integrated protons are now from $10^{20}$ to $10^{21}$.

These considerations have naturally led to the development of hardware and control systems to both greatly minimize the potential for large accidental beam loss, and to enhance primary transport and targeting precision. While significant differences exist in a number of design constraints for each beam system, many common features have been incorporated into the designs for these highest power external proton beams. All have developed comprehensive beam permit or interlock systems to guard against mis-steered high intensity beam. Accelerator and proton transport conditions are assessed for each beam pulse prior to a decision to enable extraction This is then repeated for millions of beam pulses. Precision transport and targeting control is needed both to minimize dynamic stress effects on targets, and for physics background considerations. Machine quality vacuum levels are maintained in these single pass beam transports to eliminate the beam loss from vacuum interface windows. Beam loss monitors are used extensively to quantify any lost beam, and as inputs into the permit protection systems. Beam position excursions are also carefully monitored and controlled.

\section{The First Generation "Super Beams"}

The current generation of conventional type neutrino beams, produced from pion and kaon decays, have been described as "Super Beams", as although the neutrino production approach is one in use for many years, the unprecendented targeted proton beam powers make the construction and operation of these beams significantly more challenging. Described below in 
chronological start-up order are some of the design features and challenges for each of the three first generation such beams.

\subsection{NuMI (Fermilab Neutrinos at the Main Injector)}

The Fermilab NuMI beam is designed to deliver $120 \mathrm{GeV}$ protons to the graphite neutrino production target with a system design beam power of $400 \mathrm{~kW}$. The proton beam is extracted from the Main Injector with a fast single turn kicker extraction, and a pulse length of $9.6 \mu \mathrm{s}$. Operating cycle time is $2.2 \mathrm{~s}$. Typical current NuMI beam intensity is 3.0 to $3.7 \times 10^{13}$ protons per pulse, dependent on cycle sharing with other users. Operating beam power varies from 265 $\mathrm{kW}$ in shared mode to $320 \mathrm{~kW}$ in NuMI only mode.. After extraction, the proton transport line is bent downward at 156 mradians to a Pretarget beam enclosure and Target Hall, both mined in dolemitic rock. Beam size at the target is $1.1 \mathrm{~mm} \sigma$ in both planes. Final targeted proton beam angle is downward by 58 mradians providing aim of the neutrino beam toward the MINOS far detector site located $735 \mathrm{~km}$ away in Soudan, MN.

This beam was commissioned beginning in December 2004. Since the May 2005 start of operation for the MINOS $v_{\mu}$ disappearance experiment, a total of $4.9 \times 10^{20}$ protons on target have been accumulated as of June 2008 .

Severe limits on proton beam transport loss are imposed to provide environmental ground water protection as the unshielded beam tunnel passes through an underlying aquifer region. These fractional beam loss limits of a few $\times 10^{-5}$ of the beam are also well matched to levels which provide minimal component residual radioactivity.

Operation of the NuMI proton beam has been very smooth, with system uptime availability of $98 \%$ during each of the first three years. The NuMI permit system has been very effective in preventing proton beam loss, as can be seen in Figure 1, showing one month of data. Of 22 million beam pulses to date, a total of five pulses have had significant beam loss of $1 \%$ or greater. Another feature is a fully automated beam position control (Autotune), with beam centering on target maintained within $100 \mu \mathrm{m}$, without any manual beam system tuning.

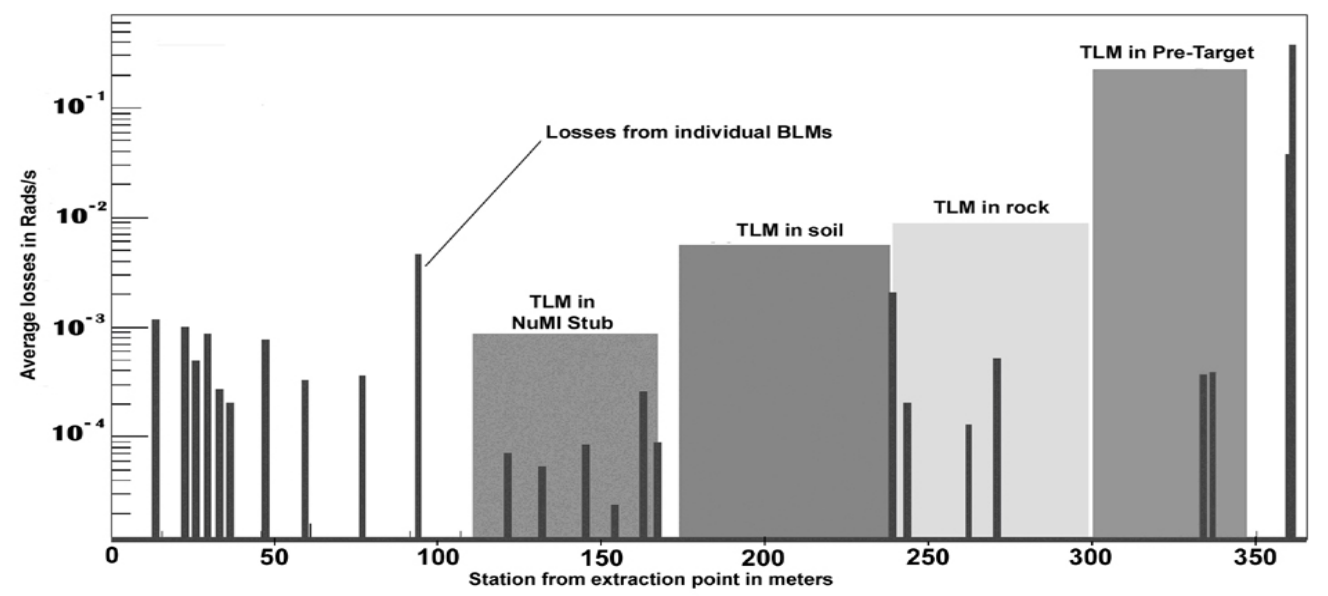

Figure 1. Beam loss along the NuMI proton line averaging for all beam extractions over a one month period. The beam loss scale can be obtained by the reading of the most downstream BLM which sees beam loss from the target profile monitor. This monitor scatters $10^{-5}$ of the beam passing through it. 


\subsection{CNGS (CERN Neutrinos to Gran Sasso)}

The CERN CNGS proton beam delivers $400 \mathrm{GeV}$ protons extracted from the SPS to the neutrino production target with a design beam power of $500 \mathrm{~kW}$. Two beam extractions separated by $50 \mathrm{~ms}$ are provided for each acceleration cycle, with cycle repetition rate of $6.0 \mathrm{~s}$. Design intensity is $2.4 \times 10^{13} \mathrm{ppp}$ for each extraction. Beam size at the target is $0.5 \mathrm{~mm}$ sigma, with a batch length of $10.5 \mu$ s. Due to the combination of intensity and higher beam energy, the CNGS beam is potentially damaging for a single mis-steered beam cycle, and requires significant attention to prevention of mis-steered beam and to targeting precision.

CNGS beam was commissioned efficiently during the summer of 2006, and is currently in the first extended data accumulation run for the Opera detector measuring $v_{\mu}$ to $v_{\tau}$ appearance.. The detector is located at Gran Sasso, Italy, $730 \mathrm{~km}$ from CERN. A total of $2.6 \times 10^{19}$ protons on target are planned for 2008 operation.

A very comprehensive beam interlock system is used to insure readiness for each CNGS beam pulse prior to extraction. The interlock system evaluates beam conditions in the SPS prior to extraction, kicker status, CNGS power converter status, target station and hadron stop readiness, plus transport line beam loss and trajectory for the previous pulse. Beam position is centered on the target to significantly less than $0.5 \mathrm{~mm}$, with inhibit of the 2 nd extraction in a cycle for a first pulse offset by greater than this tolerance. Shown in Figure 2 is the robust CNGS proton beam optics design.

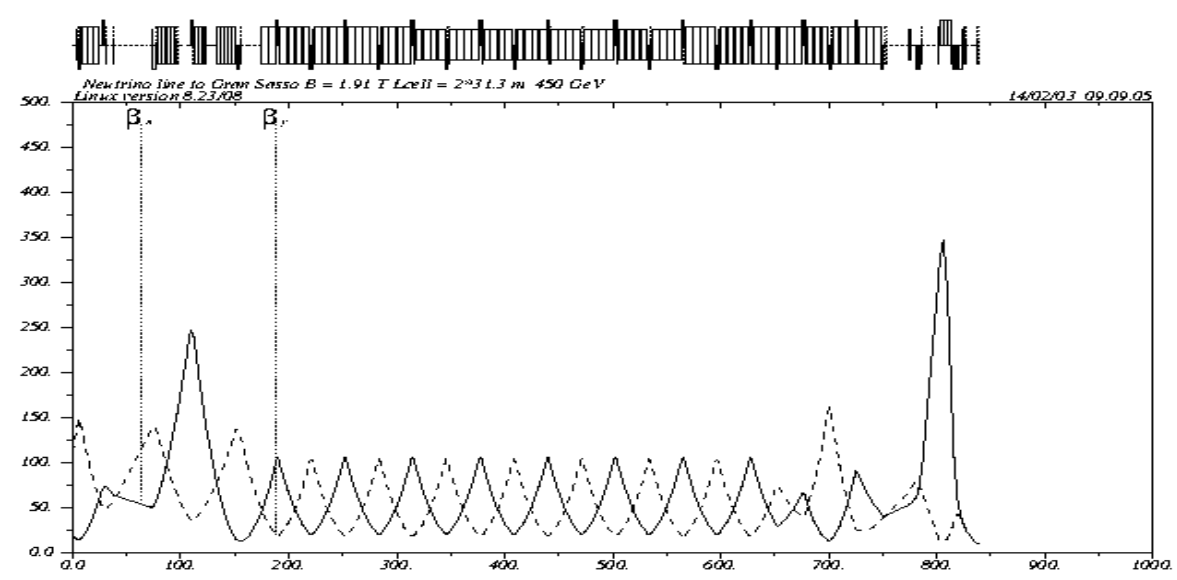

Figure 2. CNGS proton beam optics. Beta at focus in both planes $=10 \mathrm{~m}$

Sigma at $400 \mathrm{GeV}=0.5 \mathrm{~mm}$. Beam spot stability is well within $0.5 \mathrm{~mm}$ tolerance

\subsection{T2K (Tokai to Kamioka)}

The T2K long baseline $v_{\mu}$ to $v_{\mathrm{e}}$ appearance experiment will utilize a neutrino beam initially produced by targeting $30 \mathrm{GeV}$ protons from the new J-PARC accelerator complex. Slated for start-up in April 2009, the initial beam system plan is for $450 \mathrm{~kW}$, with design capability to accept future higher power beam with $50 \mathrm{GeV}$ protons. Excellent progress is currently seen with the accelerator complex commissioning.

The significant primary beam challenge for T2K is due to a long 84.5 deg.bend needed in limited space to aim the beam toward the Super-Kamiokande detector at a distance of $295 \mathrm{~km}$. This bend is accomplished by innovative design superconducting combined function magnets. 
Beam loss in the superconducting arc section must then be maintained at less than $1 \mathrm{~W} / \mathrm{m}$. Upstream of this arc region is a normal conducting magnet preparation section. Any extended beam tails for the $10 \pi \mathrm{mm} \mathrm{mr} 30 \mathrm{GeV}$ beam will be collimated in the $81 \pi \mathrm{mm} \mathrm{mr}$ admittance preparation section prior to entering the arc region of admittance $>200 \pi \mathrm{mm} \mathrm{mr}$. Modeling indicates a beam energy deposition in the superconducting arc region which is reduced by 4 orders of magnitude from that in the preparation section. A final focusing section then establishes beam targeting with a position stability requirement of $<1.0 \mathrm{~mm}$.

The $\mathrm{T} 2 \mathrm{~K}$ proton beam is also being equipped with a comprehensive set of beam instrumentaiton. As for the other high power proton beams a beam loss and beam position sensing protection system will provide decision for beam extraction on an each pulse basis. The added challenge here is the beam loss sensitivity of the superconducting magnets.

\section{Lessons for Maga-Watt Proton Beams}

As we look toward significant increases in proton beam power, an assessment is made of how the current generation super beams design and control would be applicable at much higher beam power. The fundamental approach currently used should be readily appropriate for higher power beams. Key is to do beam control as is presently done, but ever more carefully as beam power increases.

- The most important protection remains with a comprehensive and well tested beam interlock or permit system. No beam should be extracted until all parameters are at specifications within tight tolerances.

- Robust design is needed for beam optics and aperture clearance. Beam loss should be very low in normal conditions. For abnormal conditions extraction should be inhibited.

- A robust automated beam position control system can reliably maintain beam targeting to high precision. Its first mission should be to "do no harm".

- Precision monitoring of instrumentation stability is needed during high intensity operation. Inaccurate BPM readings can occur from many possible problems, and are potentially dangerous. But electrostatic pickup BPM's are the mainstay beam position monitors, making effective real time calibrations important.

\section{Acknowledgments}

Contributions from Mary Bishai, Edda Gschwendtner, Atsuko Ichikawa, Takashi Kobayashi, Kazuhiro Tanaka and Jorg Wenninger are greatly appreciated. Also, a continuing gratitude to many colleagues of CNGS, K2K, MiniBooNE, NuMI and T2K, from whom we have learned much.

This work is supported in part by Fermi Research Alliance, LLC under Contract No. DEAC02-07CH11359 with the United States Department of Energy. 